\title{
Heating Characteristics of Transformer Oil-Based Magnetic Fluids of Different Magnetic Particle Concentrations
}

\author{
A. Skumiel · T. Hornowski • A. Józefczak
}

Received: 29 March 2010 / Accepted: 16 July 2010 / Published online: 30 July 2010

(C) The Author(s) 2010. This article is published with open access at Springerlink.com

\begin{abstract}
The heating ability of mineral oil-based magnetic fluids with different magnetic particle concentrations is studied. The calorimetric measurements were carried out in an alternating magnetic field of $500 \mathrm{~A} \cdot \mathrm{m}^{-1}$ to $2500 \mathrm{~A} \cdot \mathrm{m}^{-1}$ amplitude and of $1500 \mathrm{kHz}$ frequency. The revealed $H^{n}$ law-type dependence of the temperature increase rate, $(\mathrm{d} T / \mathrm{d} t)_{t=0}$, on the amplitude of the magnetic field indicates the presence of superparamagnetic and partially ferromagnetic particles in the tested samples since $n>2$. The specific absorption rate (SAR) defined as the rate of energy absorption per unit mass increases with a decrease of the volume fraction of the dispersed phase. This can be explained by the formation of aggregates in the samples with a higher concentration of magnetic particles.
\end{abstract}

Keywords Heating effect · Hysteresis losses - Magnetic fluid - Relaxation losses · Specific absorption rate

\section{Introduction}

Magnetic fluids with high-quality mineral (or synthetic) oil as a carrier liquid and with a high concentration of magnetic particles have found application in the electric power industry, particularly in high-voltage devices (transformers, switches). Magnetic fluids have been shown to provide thermal and dielectric benefits to power transformers. They can improve the cooling by enhancing the fluid circulation within transformer windings due to a magnetically driven flow, as well as they can increase the transformer capacity to withstand lightning impulses, while minimizing the effect of moisture in

\footnotetext{
A. Skumiel $(\varangle) \cdot$ T. Hornowski · A. Józefczak

Institute of Acoustics, Faculty of Physics, Adam Mickiewicz University, Umultowska 85,

61-614 Poznań, Poland

e-mail: skumiel@amu.edu.pl
} 
typical insulating fluids [1]. The benefits of magnetic fluids may be utilized to design smaller, more efficient new transformers, or to extend the life or loading capability of existing units. However, the side effect of utilizing magnetic fluids is their heating under the influence of an alternating magnetic field, especially at high frequencies. The size of this effect was studied in this article by experimental evaluation of the magnetic fluid specific absorption rate (SAR).

The heating of a magnetic fluid is a result of absorbing energy from an alternating magnetic field and converting it into heat by means of relaxation losses accompanying demagnetization and hysteresis losses during reversal of magnetization. Also, an ac magnetic field is able to induce Foucault currents in magnetic fluids, like in the elements of a magnetic transformer core. However, this mechanism of heat release is practically negligible in the case of transformer oil-based magnetic fluids due to their very low electrical conductivity.

For magnetic fluids with a particle radius below $40 \mathrm{~nm}$, the prevailing heating mechanism involves relaxation losses. There are two distinct mechanisms by which the magnetization of a magnetic fluid may relax after removing the applied magnetic field. The Brown mechanism involves rotations of the entire magnetic particle relative to the surrounding medium, while the Néel mechanism is due to reorientation of the magnetization vector within the particle. The thermal losses corresponding to Néel and/or Brownian relaxation are proportional to the square of the magnetic field amplitude [2]. If a magnetic fluid contains magnetic particles with sizes of a few dozen nanometers or more, the magnetization exhibits a hysteresis effect which is the source of additional thermal losses. The dependence of hysteresis losses upon the magnetic field amplitude for a so-called Rayleigh loop may be well described by a third-order power law [3].

The aim of this study was a determination of the contribution of relaxation and hysteresis losses to the total thermal energy released during external magnetic field changes, for samples with different concentrations of magnetic grains. Also, the influence of the magnetite volume concentration on the specific absorption rate, $S A R_{\text {sample }}$ (defined as the thermal power dissipation in the unit mass of the sample) and on the $S A R_{\mathrm{Fe}}$ (defined as the thermal power dissipation divided by the mass of magnetic crystal content in the sample) was investigated.

\section{Materials and Methods}

The magnetic fluid, composed of transformer oil ITO 100 and $\mathrm{Fe}_{3} \mathrm{O}_{4}$ particles coated with oleic acid, was prepared in the Slovak Academy of Science (Košice) by the method described in [4]. A vibrating sample magnetometer (VSM, home-made) was used to measure the magnetization curve of the magnetic fluid studied. For a description of the dispersion of magnetite particles, the lognormal distribution is usually used [5]. From the parameters of the particle size distribution function, $d_{0}=9.48 \mathrm{~nm}$ and $\beta=0.407$, which were extracted from the magnetization curve, the mean radius, $\langle d\rangle=10.27 \mathrm{~nm}$, and standard deviation of particle size, $\sigma=4.36 \mathrm{~nm}$, were determined. The particle size distribution function $f(d)$, obtained from VSM measurements is shown in Fig. 1 [6]. 
Fig. 1 Lognormal distribution of magnetite particle diameters dispersed in mineral oil obtained from VSM measurements

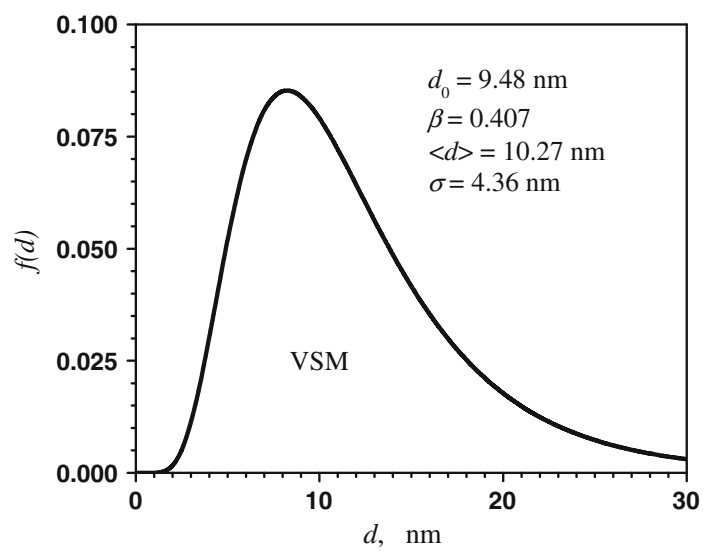

From the VSM data, using the mean-spherical and the high-temperature approximation models [7], the value of the magnetization in the saturation state was calculated as $M_{\mathrm{S}} \cong(29.19 \pm 0.03) \mathrm{kA} \cdot \mathrm{m}^{-1}$. The saturation magnetization allowed us to determine the volume fraction of the magnetite phase from the expression,

$$
\phi=\frac{M_{\mathrm{S}}}{M_{\mathrm{b}}}
$$

where $M_{\mathrm{b}}=446 \mathrm{kA} \cdot \mathrm{m}^{-1}$ is the spontaneous magnetization of magnetite grains. In the case of the magnetic liquid studied, Eq. 1 leads to a value of $6.6 \%$.

The initial magnetic fluid was then diluted with transformer oil in proportions 1:2, 1:4, and 1:8 to obtain four samples with concentrations $\phi_{1}=6.6 \%$ (non diluted), $\phi_{2}=3.3 \%, \phi_{3}=1.65 \%$, and $\phi_{4}=0.825 \%$. They were denoted as MF1, MF2, MF3, and MF4, respectively.

The specific heat capacity at constant pressure, $C_{p}$, for pure oil and the MF1 sample, was measured with the help of a DSCQ2000 calorimeter in the temperature range of $15^{\circ} \mathrm{C}$ to $45^{\circ} \mathrm{C}$. The values of the specific heat capacity for MF2, MF3, and MF4 samples were calculated according to the procedure described in [8]. Additionally, the density and viscosity of the studied samples were measured, but the details of these measurements are presented elsewhere [9]. The values of the physical parameters characterizing the magnetic fluid samples investigated in this study are listed in Table 1.

The experimental setup used for calorimetric measurements permitted a continuous recording of the temperature inside the sample upon its heating by an external alternating magnetic field. A glass tube containing the sample was thermally isolated by a layer of material from the solenoid winding supported on a plastic sleeve. The solenoid was a part of a series RLC circuit and had a length of $78 \mathrm{~mm}$ and a self-inductance of $15.6 \mu \mathrm{H}$ in air. The circuit was supplied with the sinusoidal signal with a frequency of $1500 \mathrm{kHz}$ and an adjustable amplitude. Before heating started, the temperature of the sample was set to $25^{\circ} \mathrm{C}$. 
Table 1 Values of the specific heat capacity $C_{p}$, volume concentration $\phi$, density $\rho$, mass of magnetic material per unit volume of fluid $m_{\mathrm{Fe}}$, and shear viscosity coefficient $\eta_{\mathrm{S}}$, determined at temperature $T=25^{\circ} \mathrm{C}$

\begin{tabular}{llllll}
\hline Sample & $C_{p}\left(\mathrm{~J} \cdot \mathrm{g}^{-1} \cdot \mathrm{K}^{-1}\right)$ & $\phi(\%)$ & $\rho\left(\mathrm{g} \cdot \mathrm{cm}^{-3}\right)$ & $m_{\mathrm{Fe}}\left(\mathrm{g}_{\mathrm{Fe}} \cdot \mathrm{cm}^{-3}\right)$ & $\eta_{\mathrm{S}}(\mathrm{mPa} \cdot \mathrm{s})$ \\
\hline MF1 & $1.2834 \pm 0.0001$ & $6.600 \pm 0.005$ & $1.6635 \pm 0.0001$ & $0.3367 \pm 0.0002$ & - \\
MF2 & $1.4851 \pm 0.0001$ & $3.300 \pm 0.005$ & $1.1905 \pm 0.0001$ & $0.1683 \pm 0.0002$ & $27.1 \pm 0.01$ \\
MF3 & $1.6495 \pm 0.0001$ & $1.650 \pm 0.005$ & $1.0125 \pm 0.0001$ & $0.0842 \pm 0.0002$ & $14.28 \pm 0.01$ \\
MF4 & $1.7545 \pm 0.0001$ & $0.825 \pm 0.005$ & $0.9237 \pm 0.0001$ & $0.04209 \pm 0.0002$ & $11.50 \pm 0.01$ \\
Mineral oil & $1.8795 \pm 0.0001$ & 0 & $0.8506 \pm 0.0001$ & 0 & $9.61 \pm 0.01$ \\
\hline
\end{tabular}

The temperature of the sample was controlled using a thermocouple (LT Lutron TM-917) with an uncertainty of $0.1{ }^{\circ} \mathrm{C}$. A detailed description of the experimental setup for calorimetric measurements can be found in $[10,11]$.

\section{Results and Discussion}

Figure 2 shows an exemplary plot of the time-dependent temperature rise of the MF2 sample for different values of the alternating magnetic field at a frequency of $1500 \mathrm{kHz}$. As is seen from the figure for small heating times, the temperature increase is roughly linear, but over longer period of heating, the heat released from the sample to its environment begins to play a role. Thus, only the initial slope of the time-temperature curve is a measure of the power release in a unit volume of the magnetic fluid.

For single-domain superparamagnetic particles, thermal energy losses in an ac magnetic field result from a relaxation process. A characteristic feature of this phenomenon is that the volumetric power dissipation is proportional to the square of the magnetic field amplitude, $H$, [2];

$$
P=\pi \mu_{0} \chi_{0} f H^{2} \frac{2 \pi f \tau}{1+(2 \pi f \tau)^{2}},
$$

Fig. 2 Time-temperature curves of magnetic fluid sample MF2 for different values of ac magnetic field of frequency $f=1500 \mathrm{kHz}$

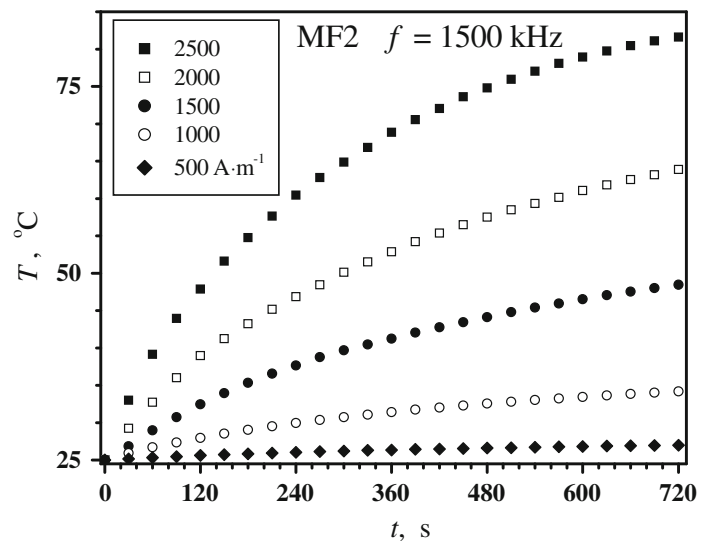


where $\chi_{0}$ is the equilibrium susceptibility of a magnetic fluid, $f$ is the frequency of the alternating magnetic field, $\tau$ is the relaxation time, and $\mu_{0}=4 \pi \times 10^{-7} \mathrm{~T} \cdot \mathrm{m} \cdot \mathrm{A}^{-1}$ is the permeability of free space.

The relaxation process in magnetic fluids proceeds according to two independent mechanisms. In Brownian relaxation, a whole particle rotates together with the vector of spontaneous magnetization. This rotation is resisted by a viscous torque due to the surrounding carrier liquid and is described by a relaxation time $\tau_{\mathrm{B}}$ which depends on the hydrodynamic volume $V_{\mathrm{h}}$, shear viscosity $\eta_{\mathrm{S}}$, and temperature $T$,

$$
\tau_{\mathrm{B}}=\frac{3 \eta_{\mathrm{S}} V_{\mathrm{h}}}{k_{\mathrm{B}} T}
$$

The hydrodynamic volume depends on the hydrodynamic radius $r_{\mathrm{h}}$ which is larger than the magnetic particle radius $r_{\mathrm{m}}$ by a thickness $\delta$ of a surfactant layer preventing coagulation of particles. In the Néel mechanism of relaxation, the magnetic moment may reverse direction within a particle by overcoming an energy barrier equal to $K V_{\mathrm{m}}$, where $K$ is the anisotropy constant of the magnetic material and $V_{\mathrm{m}}$ is the particle volume. A characteristic time associated with Néel relaxation is given by

$$
\tau_{\mathrm{N}}=\tau_{0} \exp \left(\frac{K V_{\mathrm{m}}}{k_{\mathrm{B}} T}\right)
$$

with $\tau_{0} \approx 10^{-9} \mathrm{~s}$. When both mechanisms act simultaneously, the effective magnetic relaxation time $\tau_{\text {eff }}$ of the magnetic fluid is given by

$$
\tau_{\text {eff }}=\frac{\tau_{\mathrm{N}} \tau_{\mathrm{B}}}{\tau_{\mathrm{N}}+\tau_{\mathrm{B}}}
$$

The mechanism with the shortest relaxation time is dominant. To achieve high heating rates, the Néel relaxation must not be allowed to dominate. Figure 3 shows the plot of relaxation time constants versus particle size for magnetite particles suspended in the transformer oil. As seen from the figure, both mechanisms give equal contributions to magnetization for a magnetic radius of $7.6 \mathrm{~nm}$ which is comparable with a mean radius of magnetite particles in our systems. Also, the contribution of relaxation losses to the total power losses is far from a maximum since for $f=1500 \mathrm{kHz}, \omega \tau_{\text {eff }} \approx 100$.

The relaxation losses (Brown and/or Néel) are features of small single-domain magnetic particles. However, in polydispersive systems, there is a fraction of particles significantly larger than the mean size (Fig. 1) and they are responsible for hysteresis losses proportional to the third power of the amplitude of the magnetic field. In order to check their contribution to the total power loss in the samples with different concentrations of magnetite particles, the experimental values of the heating rate $(\Delta T / \Delta t)_{t=0}$ were plotted in Fig. 4 as a function of the alternate magnetic field amplitude. Solid lines show the fit of the function $(H / a)^{n}$ to the experimental data. The experimental data used to plot the graph in Fig. 4 are listed in Table 2, and the values of the fitting parameters $a$ and $n$ are listed in Table 3 .

The value of $n$ for each sample is larger than two which indicates the simultaneous presence of relaxation and hysteresis energy losses. Assuming energy additivity, the 
Fig. 3 Time constants versus particle size radius for magnetite particles immersed in mineral transformer oil

Fig. 4 Experimental values of heating rate $(\Delta T / \Delta t)_{t=0}$ as a function of the ac magnetic field amplitude (at $f=1500 \mathrm{kHz}$ ) for the transformer oil-based magnetic fluid samples with different concentrations of magnetite particles. Solid lines show the fit of the function $(H / a)^{n}$ to the experimental data
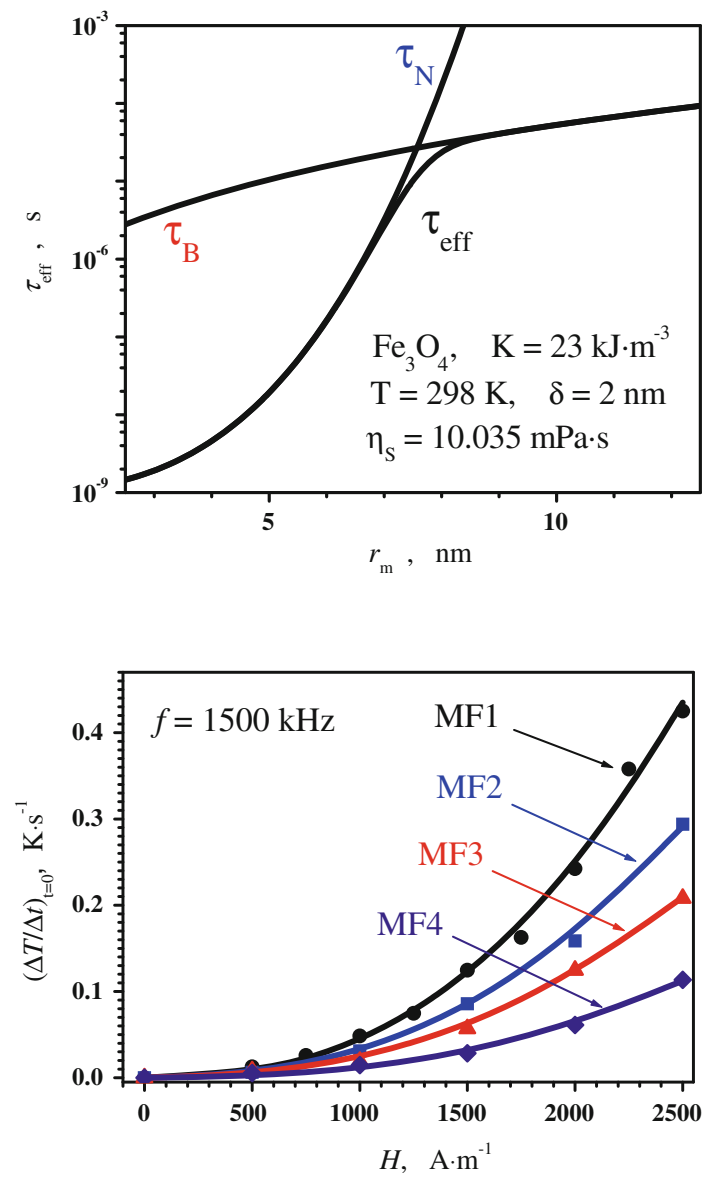

Table 2 Experimental values of the initial slope of the temperature increase rate $(\mathrm{d} T / \mathrm{d} t)_{t=0}$ for different values of the magnetic field amplitude

\begin{tabular}{lllll}
\hline$H\left(\mathrm{~A} \cdot \mathrm{m}^{-1}\right)$ & \multicolumn{4}{l}{$(\mathrm{d} T / \mathrm{d} t)_{t=0}\left(\mathrm{~K} \cdot \mathrm{s}^{-1}\right)$} \\
\cline { 2 - 5 } & $\mathrm{MF} 1$ & $\mathrm{MF} 2$ & $\mathrm{MF} 3$ & $\mathrm{MF} 4$ \\
\hline 500 & 0.01274 & 0.00637 & 0.008 & 0.00544 \\
750 & 0.02584 & - & - & - \\
1000 & 0.0485 & 0.03107 & 0.019 & 0.0147 \\
1250 & 0.0747 & - & - & - \\
1500 & 0.1247 & 0.08573 & 0.05695 & 0.0283 \\
1750 & 0.1626 & - & - & - \\
2000 & 0.2424 & 0.1586 & 0.1247 & 0.0611 \\
2250 & 0.3579 & - & - & - \\
2500 & 0.425 & 0.2938 & 0.208 & 0.1134 \\
\hline
\end{tabular}


Table 3 Values of the parameters obtained from fitting Eq. 6 to the experimental data

\begin{tabular}{llllll}
\hline Sample & $a$ & $n$ & $r$ & $h$ & $H_{0}\left(\mathrm{~A} \cdot \mathrm{m}^{-1}\right)$ \\
\hline MF1 & $3486 \pm 73$ & $2.5 \pm 0.1$ & $5604 \pm 34$ & $4057 \pm 14$ & $2126 \pm 48$ \\
MF2 & $4531 \pm 115$ & $2.5 \pm 0.1$ & $6941 \pm 33$ & $5453 \pm 14$ & $5453 \pm 58$ \\
MF3 & $4713 \pm 124$ & $2.5 \pm 0.1$ & $5873 \pm 71$ & $6565 \pm 29$ & $8206 \pm 307$ \\
MF4 & $5809 \pm 329$ & $2.6 \pm 0.2$ & $8587 \pm 141$ & $7129 \pm 25$ & $4915 \pm 213$ \\
\hline
\end{tabular}

power losses, expressed as the initial slope of the heating rate $(\mathrm{d} T / \mathrm{d} t)_{t=0}$, can be written as the sum of two components:

$$
\left(\frac{\mathrm{d} T}{\mathrm{~d} t}\right)_{t=0}=\left(\frac{H}{a}\right)^{n}=\left(\frac{H}{r}\right)^{2}+\left(\frac{H}{h}\right)^{3},
$$

where $r$ and $h$ are the parameters describing relaxation and hysteresis losses, respectively. The values of $r$ and $h$, that were obtained by fitting the function given in Eq. 6 to the experimental data of the heating rate $(\mathrm{d} T / \mathrm{d} t)_{t=0}$, are listed in Table 3 .

From Eq. 6, it follows that the value of the magnetic field intensity $H_{0}$, at which both components make an equal contribution to the power loss in the magnetic field sample, amounts to

$$
H_{0}=\frac{h^{3}}{r^{2}}
$$

For $H>H_{0}$, the losses related to magnetic hysteresis definitely start dominating in the total energy losses. The values of $H_{0}$ for the magnetic fluids studied are listed in Table 3.

Figure 5 shows the experimental values of the heating rate $(\Delta T / \Delta t)_{t=0}$ as a function of the magnetite particle concentration for different magnetic field amplitudes. It can be seen that for all magnetic field amplitudes the heating rate $(\Delta T / \Delta t)_{t=0}$ increases with the magnetite volume fraction $\phi$. This finding is consistent with the results from other studies [12].

Fig. 5 Experimental values of $(\Delta T / \Delta t)_{t=0}$ as a function of the magnetite particles volume concentration for different magnetic field amplitudes

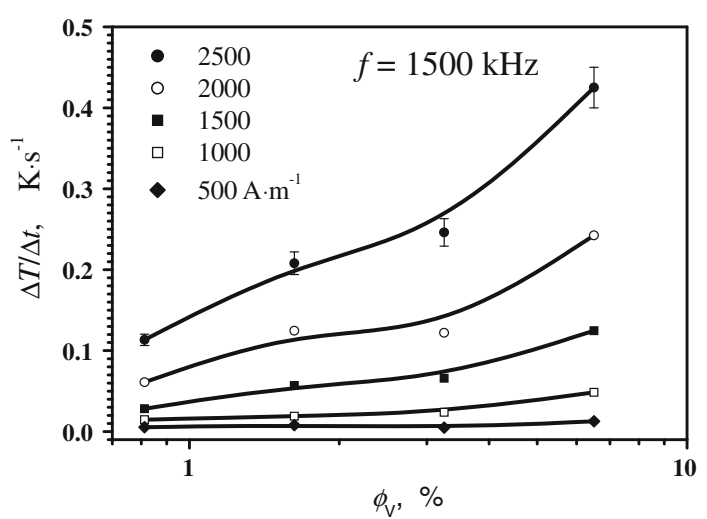


Fig. 6 Specific absorption rate referenced to a unit mass of the sample for transformer oil-based MF samples MF1, MF2, MF3, and MF4 of different concentrations of magnetite

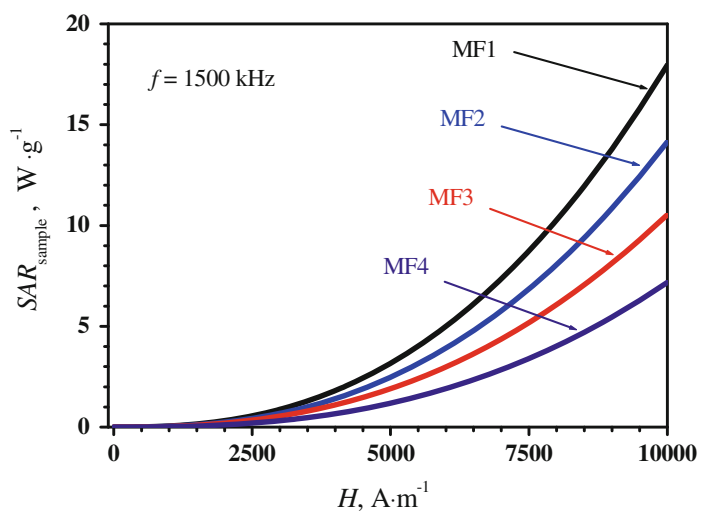

To estimate the amount of thermal power released in a unit mass of the sample, the specific absorption rate $(S A R)$ is often used, which is defined by the following formula:

$$
S A R_{\text {sample }}=C_{p}\left(\frac{\Delta T}{\Delta t}\right)_{t=0},
$$

where $C_{p}$ is the specific heat of the sample. Figure 6 shows $S A R_{\text {sample values refer- }}$ enced to a unit mass of MF1, MF2, MF3, and MF4 samples. It can be seen that for all samples, $S A R_{\text {sample values grow monotonically with an increase of the magnetic }}$ field amplitude: the higher the magnetite volume fraction $\phi$, the more pronounced is the increase of $S A R_{\text {sample. }}$.

On the other hand, a specific absorption rate $\left(S A R_{\mathrm{Fe}}\right)$, defined as the thermal power dissipation divided by the mass of a magnetic crystal $m_{\mathrm{Fe}}$, can be expressed as [13]

$$
S A R_{\mathrm{Fe}}=\frac{C_{p} \rho}{m_{\mathrm{Fe}}} \cdot \frac{\mathrm{d} T}{\mathrm{~d} t} .
$$

The $S A R_{\mathrm{Fe}}$ values as a function of the magnetic field amplitude for all samples studied are shown in Fig. 7. As seen from the figure, the samples with lower magnetite concentrations $\phi$ exhibit higher $S A R_{\mathrm{Fe}}$ values, as opposed to the $S A R_{\text {sample values }}$ shown in Fig. 6. This can be explained by the aggregate formation in the samples with higher volume concentrations of magnetite grains. Their larger volume aggregates has longer Brownian relaxation times and contribute less to the thermal energy losses. This conclusion is confirmed by the smallest value of the magnetic field amplitude $H_{0}$ for an MF1 sample.

\section{Summary}

In this study, the heating ability of transformer oil-based magnetic fluids with different concentrations of magnetite particles was investigated. The revealed $H^{n}$-law-type dependence of the temperature increase rate, $(\mathrm{d} T / \mathrm{d} t)_{t=0}$, on the amplitude of the 
Fig. 7 Specific absorption rate functions referenced to a unit mass of the magnetic material for transformer oil-based MF samples MF1, MF2, MF3, and MF4 of different concentrations of magnetite

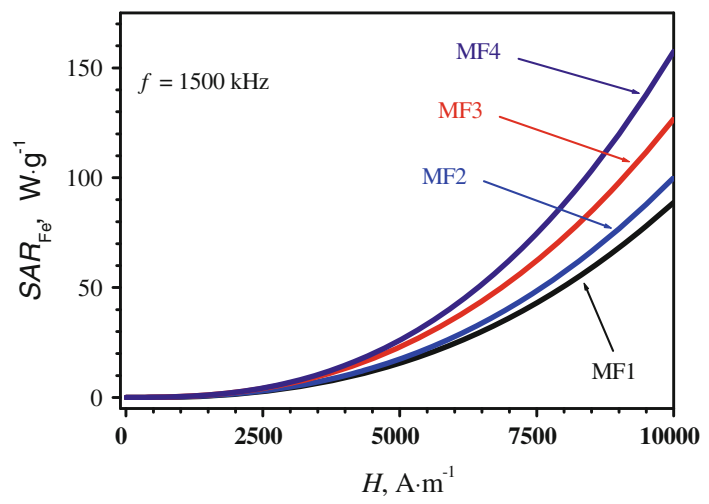

magnetic field indicates the presence of both superparamagnetic and partially ferromagnetic particles in all fluids studied since $n>2$. For superparamagnetic particles, the heating mechanism is associated with Brown and/or Néel relaxation. However, due to the presence of a small amount of ferromagnetic particles in the magnetic fluids studied in our research, the nonlinear hysteresis losses also have to be taken into account. For the lower values of the magnetic field amplitude, $H<H_{0}$, the relaxation losses dominate whereas for the opposite case, $H>H_{0}$, the magnetic hysteresis is the main source of thermal energy losses. The heating process is the most effective for samples with the biggest magnetite volume fraction. The specific absorption rate referenced to a unit mass of the magnetic material reveals a higher value for samples with a lower magnetite volume fraction. This can be explained by the formation of aggregates in more concentrated magnetic fluids.

Acknowledgments The studies were supported by the Polish Ministry of Science and Higher Education grant N202 097 32/2406, and by the Project for Polish-Slovak Bilateral Cooperation No. SK-PL-006909/8158/2010. The authors gratefully acknowledge Professor Milan Timko from the Slovak Academy of Science (Košice) for samples of magnetic fluid.

Open Access This article is distributed under the terms of the Creative Commons Attribution Noncommercial License which permits any noncommercial use, distribution, and reproduction in any medium, provided the original author(s) and source are credited.

\section{References}

1. P. Kopčanský, K. Marton, L. Tomčo, M. Koneracká, M. Timko, I. Potočová, F. Herchl, in Proceedings of the Joint 15th Riga and 6th Pamir International Conference on Fundamental and Applied MHD, vol. 1 (2005), p. 329

2. R.E. Rosensweig, J. Magn. Magn. Mater. 252, 370 (2002)

3. R. Hergt, R. Hiergeist, M. Zeisberger, D. Schüller, U. Heyen, I. Hilger, W.A. Kaiser, J. Magn. Magn. Mater. 293, 80 (2005)

4. F. Herchl, P. Kopčanský, M. Timko, M. Koneracká, K. Marton, I. Kolcunová, L. Tomčo, Acta Phys. Pol. A 113, 569 (2008)

5. M. Raşa, Eur. Phys. J. E 2, 265 (2000) 
6. M. Timko, A. Dzarova, J. Kovac, A. Skumiel, A. Józefczak, T. Hornowski, H. Gojżewski, V. Zavisova, M. Koneracka, A. Sprincova, O. Strbak, P. Kopcansky, N. Tomasovicova, J. Magn. Magn. Mater. 321, 1521 (2009)

7. A.F. Pshenichnikov, J. Magn. Magn. Mater. 145, 319 (1995)

8. L.L. Lao, R.V. Ramanujan, J. Mater. Sci. Mater. Med. 15, 1061 (2004)

9. K. Charaziak, M. Marecki, T. Hornowski, A. Józefczak, A. Skumiel, Arch. Acoust. 33, 105 (2008)

10. A. Skumiel, J. Magn. Magn. Mater. 307, 85 (2006)

11. A. Skumiel, A. Józefczak, T. Hornowski, J. Phys. Conf. Ser. 149, 012111 (2009)

12. A. Halbreich, J. Roger, J.N. Pons, D. Geldwerth, M.F. Da Silva, M. Roudier, J.C. Bacri, Biochimie 80, 379 (1998)

13. A. Skumiel, A. Józefczak, A. Szlaferek, W. Kowalski, M. Timko, P. Kopcansky, M. Koneracka, Magnetohydrodynamics 44, 191 (2008) 